\title{
Marin Palaversa
}

E-mail: marin.palaversa@fsb.hr

Pero Prebeg

E-mail: pero.prebeg@fsb.hr

\section{Jerolim Andrić}

E-mail: jerolim.andric@fssb.hr

University of Zagreb, Faculty of Mechanical Engineering and Naval Architecture

Ulica Ivana Lučića 5, 10000 Zagreb, Croatia

\section{Current State of Development of Ship Structural Design and Optimization Methods}

\begin{abstract}
This paper presents state-of-the-art methodologies and methods used in the rationally-based structural design of ships and offshore structures, namely design support system, structural optimization, surrogate modelling and sensitivity analysis. It demonstrates their application in structural design of a platform support vessel. It ends with a list of benefits that a structural designer may expect when the presented methods/methodologies are used. It also shows the obstacles to their full implementation in the engineering practice.
\end{abstract}

Keywords: structural optimization, structural design, offshore structures

\section{Introduction}

Every technical product can be seen as a system, i.e. an assemblage of individual subsystems/parts that interact with each other to exhibit a desired behaviour. Each of the subsystems can be described by a number of its properties/descriptors (e.g. material, dimensions, function, etc.). During a typical product design process, the product designer tries to find a set of the descriptors that leads to the fulfilment of requirements imposed on the product by a customer (management, buyer, etc.), producer (builder), regulatory bodies and, possibly, additional stakeholders. In order to find such a set, the designer must have relevant information about effects of the descriptors' changes on the desired product's properties (e.g. how the selection of a material influences product stiffness, weight, aesthetics, etc.). Additionally, he usually must prove the fulfilment 
of the requirements, especially those imposed by the regulation authorities (e.g. that the product's structure can withstand all loads that might occur during its lifetime).

If we think of a ship or an offshore unit as a system (product) and its structure as a subsystem [1] described by hundreds or even thousands of design descriptors/ variables (e.g. plate thicknesses, girders' and stiffeners' layout and characteristics, material properties...), the extent of a structural design problem becomes obvious. Traditionally, naval architects used to develop new designs based on similar previous designs, on their experience or, most often, by combining both of them. Once a design variant was obtained, its descriptors' values were checked against the imposed criteria (e.g. classification societies' rules) and, if all criteria were fulfilled, the design process finished proceeding to the production. Although such a design procedure (usually designated as a rulebook design [1]) is still very useful (and in many cases preferred), it has a few very important disadvantages. First, a designer most often cannot quantify the influence of a variable change on a certain design requirement (e.g. if the thickness of plating is changed, what happens to the fulfilment of limit stress criteria). Also, because a functional relationship between the input (design variables) and the output (design criteria) is not established, the designer cannot examine different variants and thus select the better one. Second, once a rulebook design procedure has finished, the resulting structure, although fulfilling all the imposed criteria, most probably does not fulfil them in the best possible way, e.g. it uses more material than needed or its safety against failure is not as high as it could be. To overcome these disadvantages a new design procedure, called rationally-based design, was formulated [2]. It is "entirely based on structural theory and computer-based methods of structural analysis and optimisation to achieve an optimum structure based on a designer-selected measure of merit" [1]. Thus, it is especially applicable in the design of offshore structures because the direct strength calculation methods, e.g. the finite element method (FEM), have been widely used in their design from the very beginning, partly because of the lack of previous experience to rely on and partly because the economic stakes and risks in case of failure are high [1].

The aim of this paper is to introduce a reader to the main elements of the rationallybased design methodology and demonstrate how it can be applied in practice. Thus, in the next chapter we give a brief overview of the theory behind those elements of the rationally-based design that a reader, not primarily involved in design methods, is less familiarised with, namely design support system (DeSS), surrogate modelling, sensitivity analysis and structural optimisation. In Chapter 3 a case study from the offshore industry demonstrates the application of these elements in the structural design of a platform support vessel [3]. At the end, we sum up with a discussion on the main benefits of the abovementioned methodology as well as some challenges that a structural designer might encounter if he decides to use it. 


\section{Methods}

\subsection{Design support system (DeSS)}

The cornerstone of the rationally-based design methodology defined in the Introduction is rational decision making. As stated in [4] "during rational decision making, individuals will survey alternatives, evaluate consequences from each alternative and finally do what they believe has the best consequences... The keys to a decision are the quality of information about alternatives and individual preferences." Based on this we can state that a designer, in order to make a rational decision, must posses the following: relevant information about the design problem (DP), a model that relates design alternative's descriptors (design variables) and the consequences of its change/s to the design criteria, a defined measure of merit (objective function) that represents the basis for the selection of one alternative (design variant) over the other and a procedure (e.g. optimisation algorithm) that can help the designer in reaching "the best" design variant. Such a methodology, applicable to the structural design problem, is called design support system (DeSS). It is described in detail in [5] and demonstrated in [6]. In the sequel its main modules will be defined and they interaction explained.

The DeSS comprises two modules (Figure 1): an analysis module (AM) and a synthesis module (SM). The AM consists of the following submodules:

- structural definition $(\Phi)$ - contains structural descriptors (geometry of structure, scantlings, materials, etc.)

- structural loads $(\varepsilon)$ - contains all the prescribed loads (e.g. by the classification societies) and/or loads calculated by a direct method (e.g. CFD), model testing, etc.

- structural response $(\rho)$ - contains information such as structural displacements, stresses, strains, etc.

- structural adequacy $(\alpha / \pi)$ - contains information about satisfaction of criteria prescribed by the regulatory bodies (e.g. limit stresses for different failure modes)

- quality/KPI (key performance indicators) $\Omega$ - contains information about some additional criteria specified by the designer (e.g. building cost, weight, ship stability, etc.).

One can notice that submodules $\Phi, \varepsilon$ and $\rho$ can be executed on a commercial FEA software, e.g. Femap/Nastran, Ansys, etc. Submodules $\alpha / \pi$ and $\Omega$ can be easily integrated with the FEA software (e.g. MAESTRO). The main characteristic of the AM as a whole is that its state is related to one and only one design variant. This means that if we change one design descriptor in submodule $\Phi$ or loading in submodule $\varepsilon$, all other submodules' states (data) change. For example, if the thickness of plating is decreased (change in $\Phi$ ), the displacements, strains and stresses increase (change in $\rho$ ), if $\varepsilon$ remains the same, which will in turn cause a change in adequacy criteria values $(\alpha)$ and quality/performance indicators $\Omega$ (e.g. structural weight will decrease). 


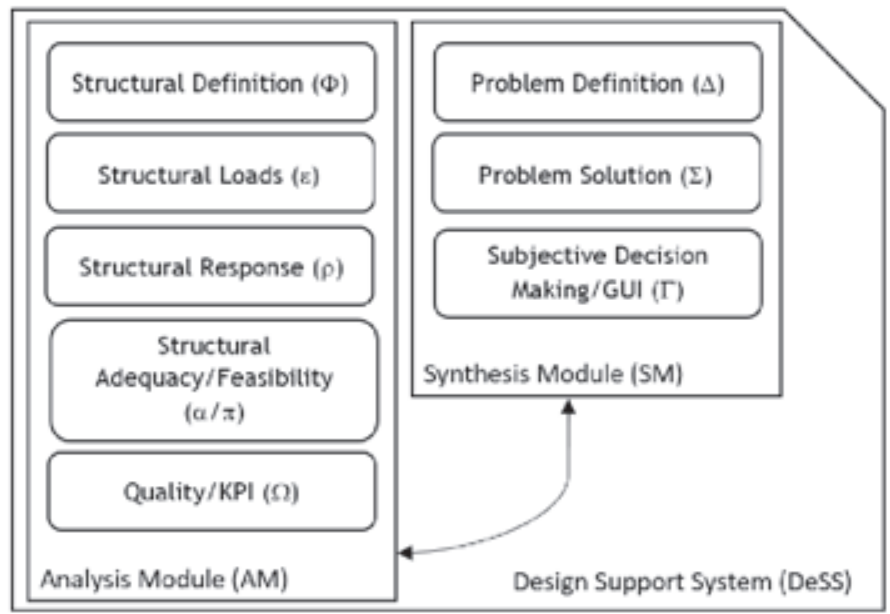

Figure 1: Scheme of the DeSS.

The SM contains following submodules:

- problem definition $(\Delta)$ where design variables (e.g. plating thicknesses, stiffeners' dimensions, girders' dimensions etc.), design constraints (e.g. limit stresses) and design objectives (e.g. minimisation of structural weight, maximisation of structural safety, minimisation of production costs, etc.) are defined

- problem solution $(\Sigma)$ where methods for the solution of the design problem are defined (e.g. optimisation algorithm)

- subjective decision making (usually with graphical user interface - GUI) $\Gamma$ where a generated Pareto front or sensitivity analysis can be graphically represented to help the designer in gaining a better insight into the design problem and make decisions (see more in [5])

When both modules are defined, we can establish a relationship between them. If we take a closer look at Figure 1, we will see that the AM is more or less the same in the rationally-based design methodology as it is in the traditional design methodology, especially if the direct strength calculation methods (e.g. FEM) are used (what has been the case in the offshore industry for decades [1]). The difference lays in the synthesis module as this role is played by the designer himself in the traditional design methodology. Thus, the AM serves as a source of information necessary for the designer to make his decisions while the design process, if we see it as a process of sequential decision making, lays in the SM module entirely. So, what is the added value of the SM module? It lays primarily in the fact that the designer himself could never find an optimal design variant among hundreds of millions of possible combinations of the design variables that all make different designs. In order to search through these design variables' combinations, we need a mathematically formulated problem that can be solved by one of the optimisation methods and this is the task of the submodule $\Sigma$. 


\subsection{Structural optimisation}

A structure in mechanics can be defined as "any assemblage of materials which is intended to sustain loads" [7]. On the other hand, to optimise means to make something optimal or best. Thus, structural optimisation is "the subject of making an assemblage of materials to sustain loads in the best way" [7]. In order to formulate a structural optimisation problem (which is closely related to the design problem described in the previous subchapter), one needs to identify (see submodule $\Delta$ in Figure 1) design variables and design criteria.

The design variables are described in the previous subchapter. The design criteria can be further divided into design constraints and design objectives. The design constraints represent limit values of the design variables. They usually come from regulatory body requirements (e.g. classification societies) which are concerned with structural safety requiring that a structure remains fit for its purpose (i.e. preventing a structural failure such as buckling, yielding, etc.). They can also come from a builder (shipyard) or an owner, e.g. in most of the shipyards it is very difficult or expensive to weld sheet plates of thickness smaller than 4 or $5 \mathrm{~mm}$ and to roll those thicker than $20 \mathrm{~mm}$ thus in an optimisation procedure these values act as constraints meaning that sheet thickness must be within these values.

As was mentioned earlier, each design variant is described by a unique set of design variables. Thus, one can form a mathematical space of design variables (called design space) where every point represents one design variant, e.g. Figure 2 shows a design space of only two design variables: $t_{p}$ which represents the thickness of plating of a ship panel and $h_{s}$ which represents the height of stiffeners on the panel.

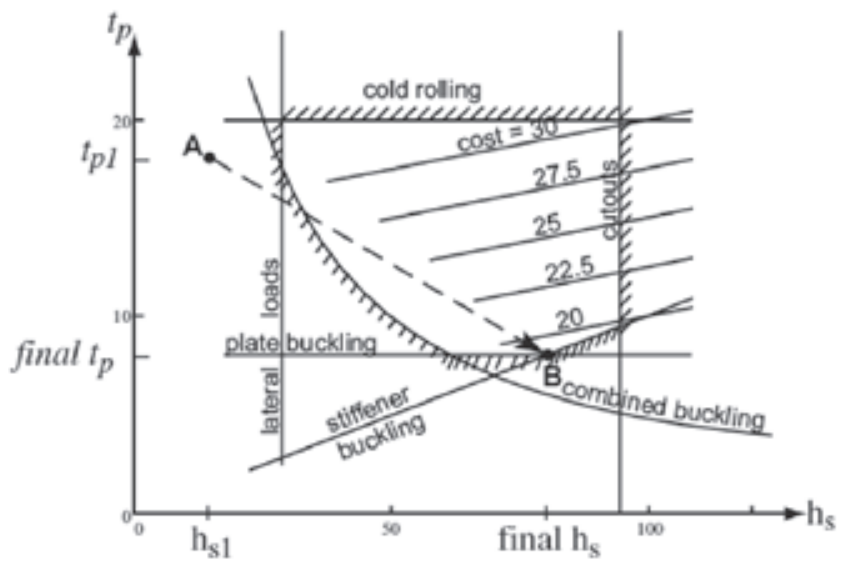

Figure 2: Example of a design space [1].

If a functional relationship is established between the design constraints and the design variables, we can depict them in the same design space. In Figure 2 they are 
depicted as curves with shaded areas. These shaded areas represent the direction in which the constraints are not met. Thus, the constraint functions bound the space of feasible designs.

And the last element of the design problem formulation are the objective functions. They are represented by contour lines in Figure 2 and represent, in this case, the total cost of the structure. As the total cost of a panel is mainly related to the amount of steel used for its fabrication, we can see that as the thickness of plating and the height of stiffeners increase, the total cost increases as well.

Once the design variables, constraints and objectives are defined and formulated in a mathematical form, an optimisation algorithm can be invoked. Its role is to, starting from the initial design point (point A in Figure 2), find a design that satisfies the objective function in the best possible manner fulfilling all the constraints. In this particular case, it means to find point $B$ which represents a design of the least cost within the area of feasible designs. In order to find the optimal design, the optimisation algorithm must search through the design space, i.e. it must invoke the whole analysis module (AM) of Figure 1 for a number of times and try to "figure out" in which direction it should proceed with its search. At this point, we will not further deal with the characteristics of different optimisation algorithms and how they manage to get from one design point to another as the aim of this subchapter is just to show the role of the optimisation algorithms in the overall design process. For a detailed study on optimisation algorithms and their characteristics, check [7] and [8]. However, it should be noted that it usually takes hundreds and sometimes even thousands of design variants to be checked [9] which means that the AM is invoked that many times. The most time consuming and computationally demanding task within the AM is the calculation of the structural response $(\rho)$ by means of the FEM. It is now obvious that if were to make hundreds or thousands of the FEA, it would make the design process so lengthy and computationally demanding that it would not fit in the time frame of a real-world structural design process. In the next subchapter, we will show how the calculation of the structural response by means of the FEM can be partly substituted for a less accurate but a much faster method.

\subsection{Surrogate modelling}

As stated in the previous subchapter, one of the most time and computationally demanding tasks within the structural design is the calculation of structural response ( $\rho$ in Figure 1) when the finite element method (FEM) is used. This is especially problematic when large (e.g. full ship) or fine mesh finite element (FE) models are used as, within the optimisation procedure, several hundreds or even several thousands of finite element analyses (response calculations) are necessary in order to obtain the optimal solution (see previous subchapter). Thus, in order to enable the use of rationally-based design methodology in engineering practice, one needs to come up 
with a different response calculation method that will be less time and computationally demanding and yet accurate enough. In the literature (e.g. International Ship and Offshore Structure Congress reports since 2003) surrogate modelling (approximate or metamodeling) has been proposed as one of such approaches.

The basic idea of surrogate modelling is to generate an approximate model that relates the design input (design variables) to the design output (design criteria) or an intermediate result (e.g. stresses) so that, instead of running numerous finite element analyses (FEA), we can make use of such simple functions (Figure 3). This modelling technique comes from the design of experiment (DoE) field and is used to establish a functional relationship between experimental input values and the observed experimental results (responses). Following are the necessary steps for generation of a surrogate model based on a number of experiments [9]:

1. planning of experiment or sampling

2. execution of simulations with original analysis methods (e.g. FEM)

3. generation or creation of the selected surrogate model

4. validation of surrogate model adequacy.

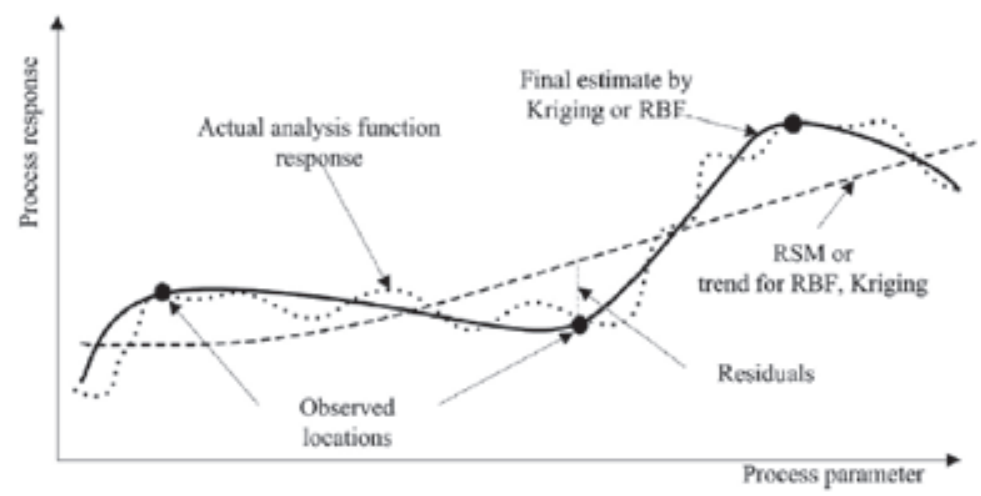

Figure 3: Example of surrogate modelling of a nonlinear response [9].

Before we start with this procedure, we need to choose experimental inputs and outputs that we want to model, i.e. establish functional relationship. In [3] this relationship was established between a number of design variables and design objective directly. This example will be analysed in detail in Chapter 3. It is important to note here that surrogate models, except for the optimisation, can be used for sensitivity analysis as well. This will be shown in the next subchapter.

Once the experimental input and output are defined, it is important to determine the number of experiments that must to be run as well as the experimental set-ups (design variables' values) so that the number of points obtained suffices the required level of model accuracy. An overview of methods that can be used for this purpose can be found in [9]. 
After that, experiments with the determined set-ups are run the required number of times. In case of structural design this means to invoke the whole analysis module (AM) of Figure 1 or only some of its submodules (e.g. if we need to establish the relationship between design variables and stress, only the $\rho$ submodule must be invoked). This step represents the most time and computationally demanding one in the design process when surrogate modelling is used.

The next step includes the selection of surrogate modelling technique as well as generation of a surrogate model. An overview of surrogate modelling techniques as well as their comparison with respect to the ship structural design problem can be found in [9].

At the end, one needs to check the accuracy of the model. This requires a few more executions of the AM with experimental set-ups that are different than those used for generation of the model in step 2. If the generated model is accurate enough, it can be used for the purpose of structural optimisation or sensitivity analysis.

\subsection{Sensitivity analysis}

Sensitivity analysis represents a study of functional value change by a change in functional argument value. Mathematically, it is based on the calculation of function's derivatives of which the first-order derivatives are usually of importance here.

Consider a function $f(\mathbf{x})$ that represents a design criterion of interest and $\mathbf{x}$ is a set of independent design variables:

$$
\mathbf{x}=\left\{x_{1}, x_{2}, \ldots, x_{p}\right\}^{T} \in \mathbb{R}^{p}
$$

then its first-order derivatives can be written as:

$$
\nabla f=\left\{\frac{\delta f}{\delta x_{1}}, \frac{\delta f}{\delta x_{2}}, \ldots, \frac{\delta f}{\delta x_{p}}\right\} \in \mathbb{R}^{p}
$$

which represents the sensitivity of $f$ with respect to $\mathbf{x}$.

Sensitivity analysis can be used by an optimisation algorithm (see subchapter 2.2) to move in the direction of greatest (or smallest) function value from the current point (gradient ascent or descent methods). Not all optimisation algorithms can use this information, but all of the mathematical programming algorithms need it in order to navigate to the optimal design (see more in [11]). 


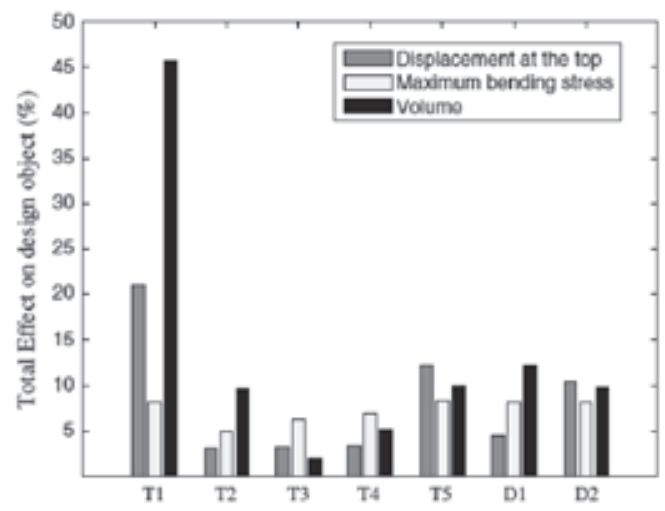

Figure 4: Sensitivity analysis of the design objectives w.r.t design variables [10].

Sensitivity analysis can also be used by a designer to get a better insight into the design problem and make decisions accordingly even if structural optimisation is not employed. Figure 4 shows the influence of different design variables from an offshore wind turbine support structural design study [10] on the design criteria. Based on this analysis the designers decided not to include design variables T2, T3 and T4 in the optimisation procedure thus reducing the size of the optimisation problem and saving time and computational resources.

The rate of change of function value per argument value (first-order derivative) can be calculated by a number of methods. One of the simplest methods is the finitedifference method. Examples of its use in sensitivity analysis as well as some other methods can be found in [11].

\section{Case study - platform supply vessel structural design}

This study is presented in [3] and all the data given in this subchapter are from the same paper.

Table 1: The main ship structural descriptors of PSV.

\begin{tabular}{|l|l|l|}
\hline Design descriptor & Value & Unit \\
\hline Length & 79.5 & $\mathrm{~m}$ \\
\hline Breadth & 18 & $\mathrm{~m}$ \\
\hline Depth (to main deck) & 8 & $\mathrm{~m}$ \\
\hline Draught & 6.7 & $\mathrm{~m}$ \\
\hline Deck and cargo rail plating thickness & 11 & $\mathrm{~mm}$ \\
\hline Bottom plating thickness & 12 & $\mathrm{~mm}$ \\
\hline
\end{tabular}




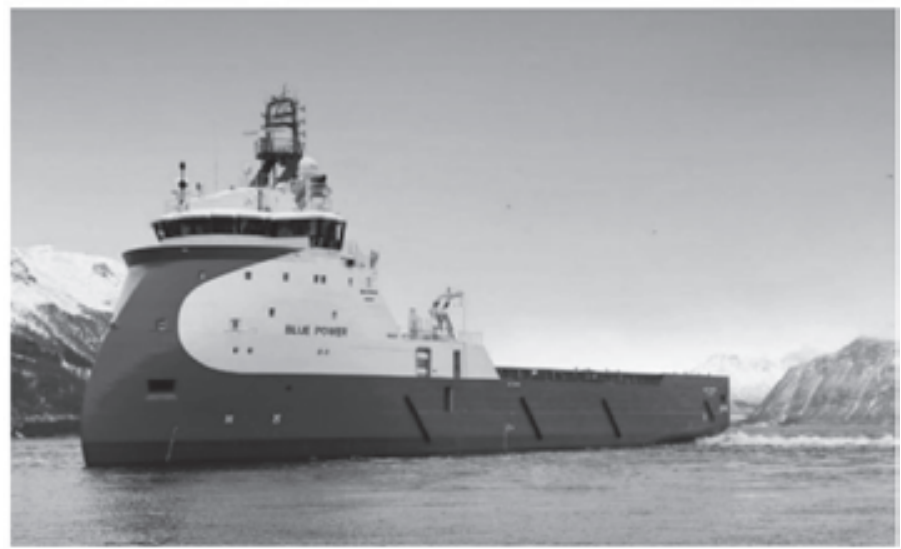

Figure 5: Platform supply vessel Ulstein PX121 [3].

The aim of the study is the structural design with optimisation of a platform supply vessel. The main vessel scantlings are given in Table 1 . The vessel can be seen in Figure 5.

The structural design procedure for the ship is shown in Figure 6. The taxonomy of modules and submodules is given according to subchapter 1.1.

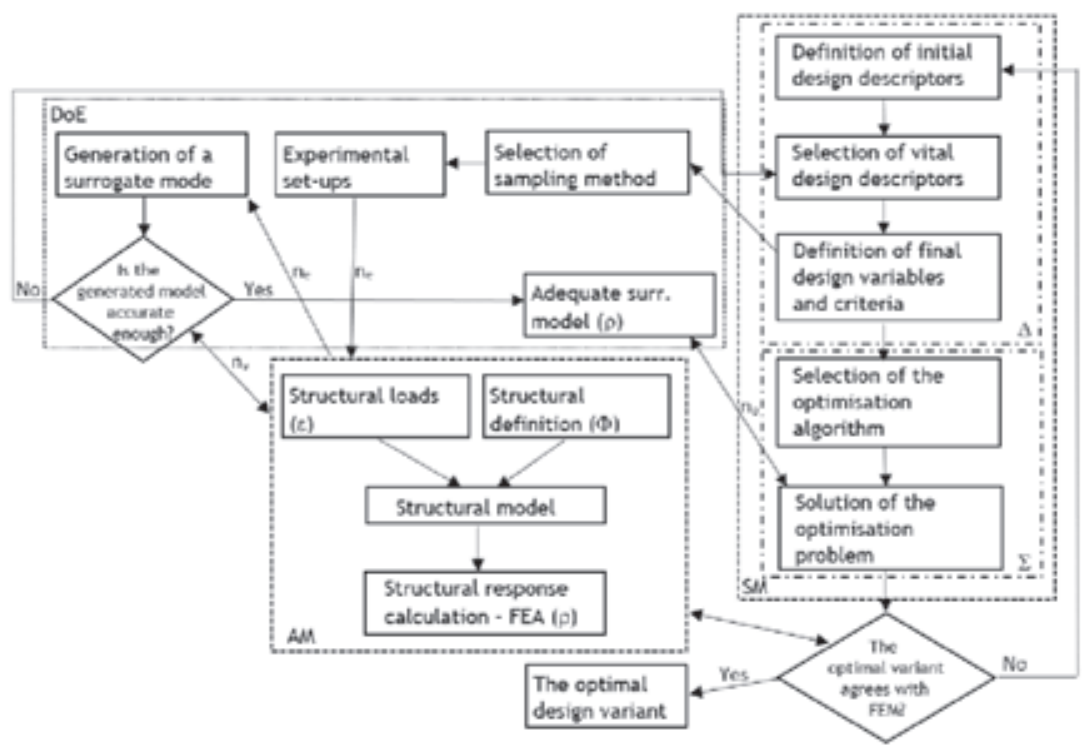

Figure 6: Structural design procedure for PSV 
The design procedure starts with the analysis of the design descriptors ("Definition of initial design descriptors" step), i.e. all the ship properties that might have an influence on ship structural design.

Among all the descriptors, it is important to select the most important ones ("Selection of vital design descriptors" step) because this leads to significant savings in time and computation, e.g. the number of experiments needed for training of a surrogate model ( $n_{e}$ in Figure 5) increases by the square of design variables [3]. This selection can be based on designer's experience, analysis of similar ships (statistics) or relevant studies (as is the case in this analysis - for more information see [3]).

After that, the final set of design variables, constraints and design objectives is selected ("Definition of final design variables and criteria" step). In this study the selected design variables are: depth $(D)$, breadth $(B)$, main deck thickness $\left(t_{\text {deck }}\right)$, bottom plate thickness $\left(t_{b o t}\right)$, cargo rail plate thickness $\left(t_{c g}\right)$, number of stiffeners $(n)$ and stiffener type $(H P)$. These structural elements can be seen in Figure 7. The design constraints are shown in Table 2. The design objectives are as follows: minimisation of structural mass $\left(R_{I}\right)$, maximisation of structural safety (i.e. minimisation of longitudinal stress in main deck $R_{2}$, bottom $R_{3}$ and cargo rail $R_{4}$ ) and maximisation of cargo hold capacity (i.e. maximisation of internal area $R_{5}$ ). If more than one objective exists, the optimisation becomes multi-objective and a number of techniques (e.g. multi-criteria decision making) exists to deal with such problems. However, in this study the concept of desirability is used to turn the multi-objective optimisation to a single-objective. Basically, if a relationship between the objectives is established (e.g. desirability of one objective over the other), then only one objective function can be obtained that combines the objectives as given by the desirability coefficients (see more in [3]).

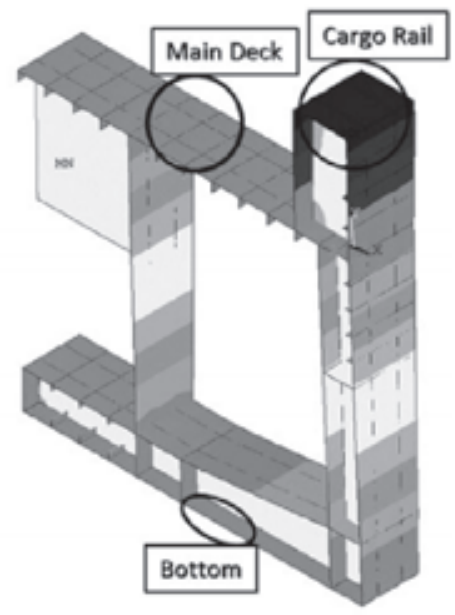

Figure 7: Cross section of ship model with stress measurement areas [3]. 
These three steps together represent a definition of the structural design problem and correspond to submodule $\Delta$ of the synthesis module (SM) as described in subchapter 2.1 .

The next submodule of the SM is the solution submodule $(\Sigma)$. It includes selection of an optimisation algorithm ("Selection of optimisation algorithm" step). In this study the simplex algorithm (based on a direct search method called Nelder-Mead method) is used. As explained in the subchapter 2.2. the optimisation algorithm finds the optimal solution by searching in the design space ("Solution of the optimisation problem" step), i.e. it must evaluate hundreds or even thousands of designs $\left(n_{o}\right)$. To make this step less computationally demanding and time consuming, the surrogate modelling is used as explained in subchapters 2.2. and 2.3. Thus, during the optimisation procedure for the response calculation, the step "Adequate surrogate model" is invoked instead of "Structural response calculation - FEA". At the end, the optimal variant is evaluated by comparing the obtained results (which are based on the surrogate model response calculations) with the results obtained from the finite element analysis.

Table 2: Design constraints.

\begin{tabular}{|l|l|l|l|}
\hline Design constraint & Min value & Max value & Unit \\
\hline Half-breadth (A) & 8 & 10 & $\mathrm{~m}$ \\
\hline Depth (B) & 7 & 9 & $\mathrm{~m}$ \\
\hline Main deck plating thickness (C) & 8 & 14 & $\mathrm{~mm}$ \\
\hline Bottom/tank top plating thickness (D) & 9 & 15 & $\mathrm{~mm}$ \\
\hline Cargo rail plating thickness (E) & 8 & 14 & $\mathrm{~mm}$ \\
\hline Stiffener number multiplier (F) & 3 & 7 & integer \\
\hline Stiffener type (G) ${ }^{1}$ & 1 & 3 & integer \\
\hline Limit stress & $/$ & 160 & $\mathrm{MPa}$ \\
\hline Internal area & 144 & $/$ & $\mathrm{m}^{2}$ \\
\hline
\end{tabular}

However, as explained in the subchapter 2.1., the SM needs data about structural responses that comes from the analysis module (AM). In this study two approaches are used: the first one is based on structural response calculation by means of the finite element method (FEM) and the other one on structural response calculation by means of a surrogate model. Therefore, two steps in Figure 6 have structural response submodule mark $(\rho)$. The steps in region "DoE" (design of experiment) follow those described in subchapter 2.3. and will not be described here again. It is important to say that in this study a method called D-optimal was used for sampling and response surfaces were

1 Numbers 1, 2 and 3 are related to the type of stiffener and correspond to symbols o-, o and o+ respectively in [6]. 
used for surrogate model generation (more on both can be found in [9] and [3]). At the end, the surrogate model is evaluated by calculating the structural response by means of the FEM in few design points different than those used for its generation. If the error is within the allowable range (defined by the designer), it is claimed to be adequate and can be used instead of the FE model for response calculation.

The following is the creation of a FE structural model, i.e. the definition of the analysis module (AM). In order to create it, we must define ship geometry and its properties (descriptors). This is usually based on the analysis of a similar ship design and is defined within the submodule $\Phi$. The loads are given by the classification societies. In this study, the critical load case is sagging. Ship structure is considered as a supported beam with bending moments at its extremes. The nodes at the extremes are related to ends of the FE model by using master-slave node relationship. This defines the ship loads ( $\varepsilon$ submodule). A detailed overview of structural model and the loads can be found in [3]. Based on this the FE structural model can be constructed (Figure 8). This model is used for the structural response calculation by means of the FEM $(\rho)$.

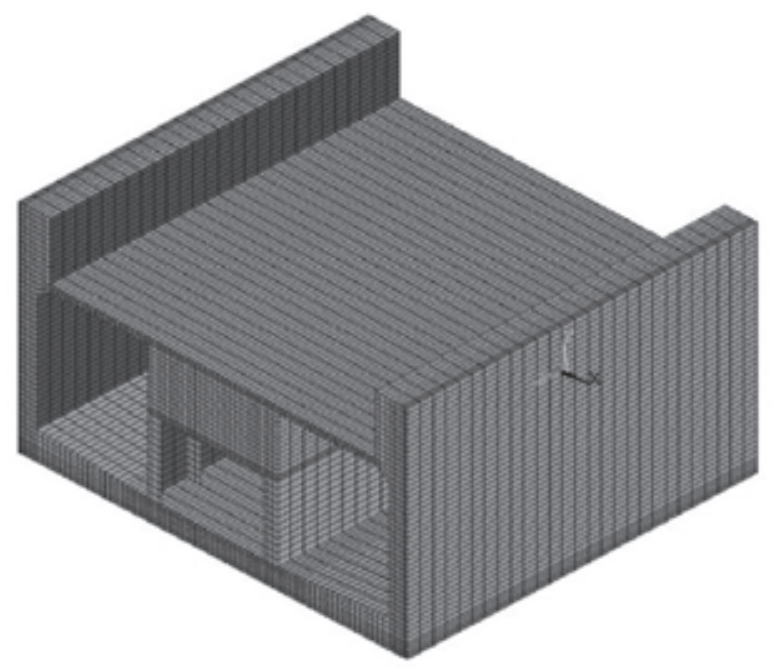

Figure 8: PSV FE structural model [6].

At the end of this rationally-based design process, a designer usually selects the best design variant and proceeds to the next design phase. However, he can use the available data to gain an additional knowledge about the design. For example, he can check whether his subjective criteria (introduced to the design thorough the desirability of one objective over the other) meet his expectations. In Table 3 the top eight most desirable design variants are shown. It can be seen that the most desirable one, when compared with the initial design (base case), has almost the same structural mass but the stresses are a bit lower and its cargo capacity (given through the internal area) is greater 
for as much as $17 \%$ (see Figure 9). This implies that the designer in this study gave the greatest desirability coefficient to the ship cargo capacity, then to the structural safety while the mass was the least important in his judgment. E.g. if the mass minimisation and structural safety were given higher desirability than the maximisation of cargo area, the solution 8 would probably be the optimum.

Based on the surrogate model, the designer can also learn more about the design. Figure 10 shows the relationship between ship breadth and cargo rail plating thickness. It can be seen that the increase in cargo rail plating thickness and ship breadth have a positive effect on design solution desirability. Figure 11 shows the relationship between ship breadth and thickness of bottom plating and its desirability function. It can be seen that the influence of ship breadth to the desirability of the design solution is much greater than that of the bottom plate thickness (again, because the designer wanted to have greater cargo are rather than smaller mass). Also, the increase in bottom plate thickness leads to a less desirable solution. From these two figures a designer can conclude that shifting the material from bottom to cargo rail is a good way to improve the design as is true for the increase in breadth.

Table 3: The eight best designs.

\begin{tabular}{|c|c|c|c|c|c|c|c|c|c|c|c|c|c|}
\hline \multirow{3}{*}{$\begin{array}{l}\text { Design } \\
\text { solution }\end{array}$} & \multicolumn{7}{|c|}{ Design variables } & \multicolumn{6}{|c|}{ Design criteria } \\
\hline & A & B & $\mathrm{C}$ & $\mathrm{D}$ & $\mathrm{E}$ & $\mathrm{F}$ & G & $\mathrm{R} 1$ & $\mathrm{R} 2$ & R3 & $\mathrm{R} 4$ & R5 & $\mathrm{De}^{2}$ \\
\hline & $\begin{array}{l}\mathrm{B} / 2 \\
(\mathrm{~m})\end{array}$ & $\begin{array}{l}\mathrm{D} \\
(\mathrm{m})\end{array}$ & $\begin{array}{l}t_{\text {deck }} \\
(\mathrm{mm})\end{array}$ & $\begin{array}{l}\mathrm{t}_{\mathrm{bot}} \\
(\mathrm{mm})\end{array}$ & $\begin{array}{l}\mathrm{t}_{\mathrm{cg}} \\
(\mathrm{mm})\end{array}$ & $\mathrm{n}$ & HP & $\begin{array}{l}\mathrm{m} \\
(\mathrm{t} / \mathrm{m})\end{array}$ & $\begin{array}{l}\mathrm{s}_{1} \\
(\mathrm{MPa})\end{array}$ & $\begin{array}{l}\mathrm{s}_{2} \\
(\mathrm{MPa})\end{array}$ & $\begin{array}{l}\mathrm{s}_{3} \\
(\mathrm{MPa})\end{array}$ & $\begin{array}{l}\mathrm{A} \\
\left(\mathrm{m}^{2}\right)\end{array}$ & \\
\hline 1 & 9.38 & 9 & 8.5 & 9.1 & 14 & 3 & 1 & 19.36 & 32.06 & 40.82 & 55.08 & 168.8 & 0.641 \\
\hline 2 & 9.37 & 9 & 8.1 & 9 & 14 & 4 & 1 & 19.47 & 31.50 & 40.56 & 54.26 & 168.7 & 0.641 \\
\hline 3 & 9.37 & 9 & 8.8 & 9 & 14 & 3 & 1 & 19.37 & 31.77 & 41.02 & 54.77 & 168.7 & 0.641 \\
\hline 4 & 9.37 & 9 & 8 & 9.3 & 14 & 3 & 1 & 19.34 & 32.40 & 40.45 & 55.40 & 168.6 & 0.640 \\
\hline 5 & 9.34 & 9 & 8.3 & 9.5 & 14 & 3 & 1 & 19.42 & 32.06 & 39.92 & 54.79 & 168.2 & 0.640 \\
\hline 6 & 9.28 & 9 & 8.5 & 9.1 & 14 & 3 & 1 & 19.28 & 31.82 & 40.63 & 54.70 & 167.0 & 0.64 \\
\hline 7 & 9.37 & 9 & 8 & 9 & 14 & 3 & 2 & 19.46 & 31.45 & 40.75 & 54.21 & 168.6 & 0.64 \\
\hline 8 & 9.27 & 9 & 8.1 & 9 & 14 & 3 & 2 & 19.39 & 31.14 & 40.59 & 53.74 & 166.9 & 0.64 \\
\hline Initial & 9 & 8 & 11 & 12 & 11 & 3 & 2 & 19.43 & 34.91 & 40.39 & 61.82 & 144 & I \\
\hline
\end{tabular}

At the end, we can also take a look at the data about the optimisation cycles $\left(n_{o}\right.$ in Figure 6) and experimental set-ups ( $n_{e}$ in Figure 6) necessary for surrogate model generation. In order to obtain the optimal solution, the optimisation software called Design Expert 8 searched through 500 design variants. On the other hand, the total of 64 design variants were evaluated in order to create the surrogate model. If we add a few more FE analyses (FEA), beside these 64, necessary during this design procedure, we get a number of approx. 100 FEA.

2 De stands for desirability which is the objective function in this study. 


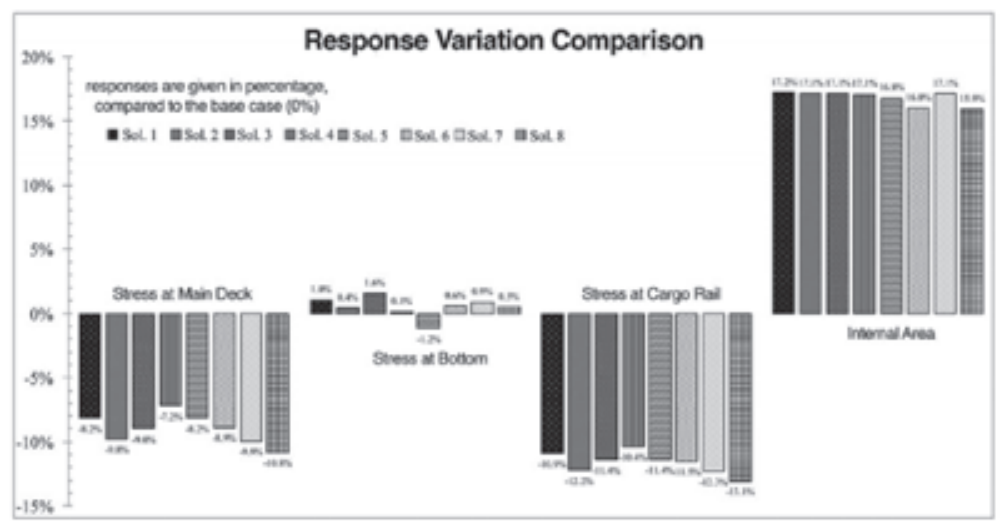

Figure 9: Response variation w.r.t the initial design [3].

If the surrogate modelling had not been used, we would have had to make approx. 400 FEA more which would have made the whole procedure much longer and computationally demanding. E.g. if the time needed for one FEA is one minute, the procedure with surrogate modelling would need approx. 100 minutes (a little bit more than an hour and a half) while the procedure without surrogate models would need approx. 400 minutes (about six and a half hours) to obtain the optimum solution. The advantage of using surrogate modelling is obvious.

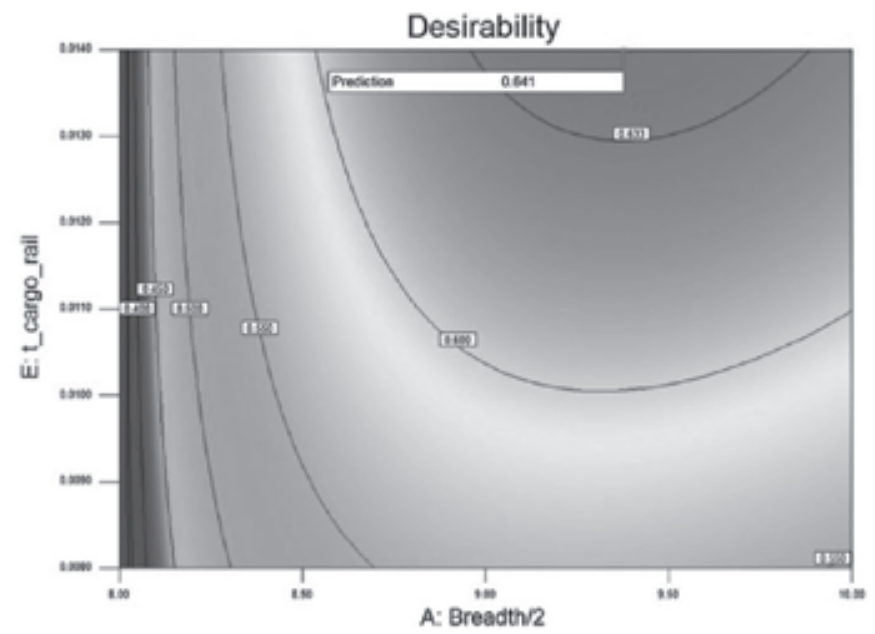

Figure 10: The combined desirability contour for cargo rail plating thickness vs. breadth based on RS surrogate model [3]. 


\section{Conclusion}

In this paper a rationally-based design has been analysed. Its main advantages over a traditional (rulebook) design have been listed, namely the direct structural analysis methods enable a direct insight into the behaviour of the structure and, when combined with optimisation methods, can lead, not only to an adequate (feasible) design, but to an optimal or "the best" design. This procedure was incorporated in a design methodology called design support system (DeSS).

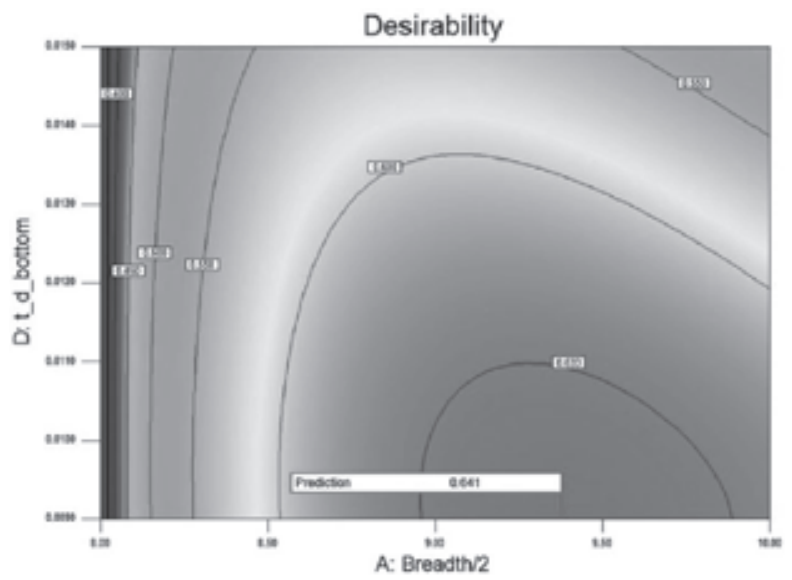

Figure 11: The combined desirability contour for bottom plating thickness vs. breadth based on RS surrogate model [3].

It has been shown that its basis represents a rational decision-making process that is based on reliable information (obtained from a DeSS module called the analysis module) and a system of decision-making incorporated in the synthesis module (SM) of the DeSS. Because the reliable information about a structural design can be obtained only by using the direct strength analysis methods, the finite element analyses (FEA) represent the cornerstone of the analysis module. Regarding the SM, its most important elements are objective and subjective decision-making methods because they enable a designer to obtain "the best" or optimal design. The objective decision-making is based on optimisation algorithms, which have been briefly explained, and an example of subjective decision-making has been given in form of design objectives' desirability defined by the designer. However, execution of optimisation algorithms (objective decision-making) means that hundreds or even thousands of design evaluations are needed and this is the primary reason why such procedures can be very lengthy and, thus, inappropriate for engineering offices. An approach has been proposed to overcome this obstacle, namely surrogate modelling. Its use, as well as the application of the whole DeSS methodology, has been demonstrated on a platform supply vessel structural design. The use of surrogate modelling in the analysed problem resulted in 
approx. 4 times less computational time than what would have been the case if only FEM had been used.

At the end, a method called sensitivity analysis has also been described and demonstrated. It can help a designer to get a better insight into the design problem by relating the change in design variables to changes in design criteria.

\section{References}

1. Hughes, O. F., Paik, J. K.: Ship Structural Design, SNAME 2010.

2. Hughes, O.F., Mistree, F., Zanic, V.: "Practical Method for the Rational Design of Ship Structures", Journal of Ship Research, Vol. 24, No.2, 101-113, SNAME 1980.

3. Andrade, S. L., Gaspar, H. M., Ehlers, S.: "Parametric structural analysis for a platform supply vessel at conceptual design phase - a sensitivity study via design of experiments", Ships and Offshore Structures, Vol. 12, 209-220, Taylor and Francis 2017.

4. Nitta, K.: "Decision making”, Encyclopaedia Britannica, Retrieved from https://www.britannica. com.

5. Zanic, V.: "Methods and concepts for the multi-criteria synthesis of ship structures", Ships and Offshore Structures, Vol. 8, 225-244, Taylor and Francis 2013.

6. Zanic, V., Andric, J., Prebeg, P.: “Design synthesis of complex ship structures”, Ships and Offshore Structures, Vol. 8, 383-403, Taylor and Francis 2013.

7. Christensen, P. W., Klarbring, A.: An Introduction to Structural Optimization, Springer 2009.

8. Coello Coello, C. A., Lamont, G. B., Van Veldhuizen, D. A.: Evolutionary algorithms for solving multi-objective problems, Springer 2007.

9. Prebeg, P., Zanic, V., Vazic, B.: “Application of a surrogate modeling to the ship structural design”, Ocean Engineering, Vol. 84, 259-272, Taylor and Francis 2013.

10. Yang, H., Zhu, Y.: "Robust design optimization of supporting structure of offshore wind turbine", Journal of Marine Science and Technology, Vol. 20, 689-702, Springer 2015.

11. Keane, A. J., Nair, P. B.: Computational Approaches for Aerospace Design - The Pursuit of Excellence, John Wiley \& Sons 2005. 
